\title{
APUNTES CONCEPTUALES PARA LA IDENTIFICACIÓN DE CONFLICTOS NORMATIVOS ENTRE NORMAS*
}

\author{
CONCEPTUAL NOTES FOR IDENTIFICATION \\ OF NORMATIVE CONFLICTS BETWEEN NORMS
}

Víctor GARCíA YZAGUIRRE*

\section{Resumen:}

El artículo tiene por objeto ofrecer criterios de identificación de conflictos normativos entre normas jurídicas. Para ello, se realiza una descripción y análisis, desde un enfoque analítico, respecto en qué casos dos normas prescriptivas condicionales son inconsistentes entre sí. Para ello $i$ ) delimitaré el tipo de normas relevantes para este estudio; ii) analizaré la necesidad de emplear nociones lógicas para conceptualizar y formular criterios de identificación de los casos de conflicto normativo, y iii) daré cuenta de las principales formas de entender la noción de conflicto normativo. Concluiré que es necesario adoptar nociones y principios de la lógica deóntica a efectos de poder diferenciar casos de antinomia y de casos que no lo son.

\section{Palabras clave:}

Conflicto normativo, normas condicionales, principio de no contradicción, lógica deóntica.

* Artículo recibido el 28 de diciembre de 2019 y aceptado para su publicación el 16 de septiembre de 2020.

** Doctorando en cotutela de la Universidad Austral de Chile y la Universidad de Génova. garciayzaguirre@gmail.com, ORCID: https://orcid.org/0000-0002-46622919 Agradezco a Álvaro Núñez Vaquero por sus comentarios y observaciones a una versión previa de este artículo. Este trabajo se enmarca como tesista del proyecto Fondecyt «Hacia una teoría del stare decisis» (núm. 1180494) concedido por el Conicyt chileno.

Problema. Anuario de Filosofía y Teoría del Derecho, núm. 15, enero-diciembre de 2021, pp. 343-371 Ciudad de México, ISSN 2007-4387, se distribuye bajo una Licencia Creative Commons ReconocimientoNo-Comercial-Sin Derivados 4.0 Internacional (CC BY-NC-ND 4.0). 


\section{VÍCTOR GARCÍA YZAGUIRRE}

\section{Abstract:}

The purpose of this article is to provide criteria for identifying regulatory conflicts between legal rules. To do so, an analytical approach is used to describe and analyze cases in which two conditional prescriptive rules are inconsistent with each other. Therefore, I will i) define the type of rules relevant to this study; ii) analyze the need to use logical constructs to conceptualize and formulate criteria for identifying cases of regulatory conflict; and iii) give an account of the main ways of understanding the concept of regulatory conflict. I conclude that it is necessary to adopt concepts and principles of deontic logic to be able to differentiate between cases of antinomy and those that are not.

\section{Keywords:}

Normative Conflict, Conditional Norms, Principle of Non-Contradiction, Deontic Logic. 
APUNTES CONCEPTUALES PARA LA IDENTIFICACIÓN...

SumARIO: I. Introducción. II. Formas de entender un conflicto normativo. III. Conclusiones. IV. Referencias.

\section{INTRODUCCIÓN}

El objetivo del presente artículo es realizar una descripción y análisis de cómo justificar la identificación de un conflicto normativo entre dos normas condicionales prescriptivas. Siguiendo a Norberto Bobbio, el estudio de los conflictos normativos está compuesto por dos preguntas teóricas diferentes: i) qué criterios nos permiten identificar que estamos frente a un conflicto normativo; y ii) qué criterios nos permiten resolver un conflicto normativo identificado. ${ }^{2}$ Mi propósito es sostener que requerimos de nociones lógicas para poder responder a la primera pregunta.

Para alcanzar este propósito realizaré lo siguiente: i) delimitaré el tipo de normas relevantes para este estudio; ii) analizaré la necesidad de emplear nociones lógicas para conceptualizar y formular criterios de identificación de los casos de conflicto normativo, y iii) daré cuenta de las principales formas de entender la noción de conflicto normativo. ${ }^{3}$

2 Bobbio, Norberto, Contribución a la teoría del derecho, Madrid, Debate, 1990, p. 339. En el mismo sentido, Lindahl, Lars y Reidhav, David, "Conflict of legal norms: Definition and varieties”, en Araszkiewicz, Michał y Płeszka, Krysztof (ed.), Logic in the Theory and Practice of Lawmaking, Cham, Springer, 2015, p. 50.

3 La pregunta sobre qué criterios nos permiten identificar que estamos frente a un conflicto normativo agrupa múltiples discusiones. Desde un punto de vista metateórico es posible detectar diversas discusiones abiertas y sumamente interesantes respecto a la conceptualización de un conflicto normativo. No pretendo dar cuenta de todas las discusiones posibles ni mucho menos entrar a detalle en estas, pero me parece oportuno indicar algunas de las más relevantes a efectos de mostrar algunos de los alcances de esta temática y con ello mostrar por qué no es posible, para los objetivos de este artículo, profundizar en todas estas. He identificado las siguientes: i) diferencias entre conflictos normativos entre normas y entre proposiciones normativas; ii) diferencias entre cómo entender un conflicto normativo por los lógicos deónticos y por los juristas que emplean la lógica deóntica; iii) diferencia entre entender un conflicto normativo como un problema genuino y quienes consideran que este es un problema aparente; iv) diferencias entre las diversas concep- 
VÍCTOR GARCÍA YZAGUIRRE

\section{FORMAS DE ENTENDER UN CONFLICTO NORMATIVO}

\section{Normas que pueden entrar en conflicto}

En este artículo sólo analizaré, por razones de espacio y precisión, cómo identificar conflictos normativos entre normas condicionales prescriptivas. Estas normas están estructuradas por un antecedente y un consecuente y son empleadas para expresar que la obligación o prohibición (de un conjunto de destinatarios) de realizar una determinada acción cada vez que se verifique una determinada circunstancia. ${ }^{4}$ Este tipo de normas son representadas formalmente de la siguiente forma: (p->0q). Bajo este esquema, el operador deóntico sólo afecta el consecuente (Oq), lo que quiere decir que el compromiso deóntico asumido es que las normas condicionales son un puente que vincula lo que es (o podría ser) un caso con lo que debe ser. $^{5}$

Las normas condicionales prescriptivas pueden ser explícitas o implícitas y podemos inferir, a partir de cada una de estas, normas

ciones disponibles de cómo entender un conflicto normativo, las cuales pueden ser agrupadas entre quienes toman el punto de vista del destinatario y quienes toman el punto de vista del legislador; $v$ ) diferencias entre las concepciones disponibles de cómo entender qué genera un conflicto normativo; vi) diferencias entre un conflicto normativo generado por normas contradictorias y un conflicto normativo generado por normas contrarias; vii) diferencias de analizar un conflicto normativo como un problema de inconsistencia o un problema de incoherencia; entre otras.

4 En términos de Alchourrón y Bulygin: «[u]na norma [jurídica] es condicional cuando la obligación, prohibición o permisión que establece está sujeta a una condición, por ejemplo, la norma que dice "Si A, entonces debe ser B". Si la obligación (prohibición, permisión) prescrita por la norma no está sujeta a ninguna condición, la norma es categórica. La gran mayoría de normas jurídicas son condicionales; ciertamente todas las normas generales lo son». Alchourrón, Carlos y Bulygin, Eugenio, "Norma Jurídica”, en Garzón Valdes, Ernesto y Laporta, Francisco (ed.), Enciclopedia Iberoamericana de Filosofía, El Derecho y la Justicia, Madrid, Trotta, 2016, p. 140.

5 Alchourrón, Carlos "Sobre derecho y lógica", Fundamentos para una Teoría General de los Deberes, Madrid, Marcial Pons, p. 144; Zuleta, Hugo, Norma y Justificación, Madrid, Marcial Pons, 2008, p. 99. Debo precisar que estoy asumiendo la concepción puente sobre las normas condicionales. 
APUNTES CONCEPTUALES PARA LA IDENTIFICACIÓN...

derivadas. Al respecto, paso a precisar cada uno de estos tipos de normas.

\section{A. Norma expresa}

Por norma explícita refiero a todos los significados atribuidos a textos normativos. Da cuenta, en este sentido, de las posibles traducciones de un texto en norma empleando un código interpretativo. ${ }^{6}$

Con esta noción se engloba a todos aquellos resultados interpretativos que han sido formulados habiéndose aplicado las reglas lingüísticas sintácticas, semánticas y pragmáticas compartidas, las distintas técnicas interpretativas en uso, y las tesis dogmáticas difundidas en doctrina. Dicho en breve, refiere a uno de los significados posibles (en un determinado espacio-tiempo en una comunidad jurídica) de un o unos textos normativos.

Una vez que hemos identificado la norma expresa, a efectos de determinar cuál es su contenido hemos de establecer qué normas podemos derivar de ella, en otros términos, qué normas derivadas justifica. ${ }^{7}$ Por normas derivadas se hace referencia a todas las normas que son inferidas mediante razonamientos lógicamente válidos, esto es, deductivos. ${ }^{8}$ La identificación de normas derivadas parte

6 Chiassoni, Pierluigi, Interpretation without Truth, a Realistic Enquiry, Cham, Springer, 2019, pp. 22 y 105. Por código interpretativo refiero, siguiendo a Chiassoni, al conjunto de reglas interpretativas compuestas por: i) reglas sobre cómo se puede traducir un texto (empleando un argumento teleológico o un argumento intencionalista, por ejemplo), y ii) reglas sobre selección de textos o reglas de preferencia entre posibles interpretaciones.

7 Cabe precisar que cada norma se implica así misma en virtud al principio de identidad normativa (Op->0p). Ratti, Giovanni Battista y Rodríguez, Jorge, "On Coherence as a Formal Property of Normative Systems”, Revus, núm. 27, 2015, p. 161.

8 Guastini, Riccardo, Interpretar y argumentar, Madrid, CEPC, 2014, p. 166. Ratti, Giovanni Battista, "An Analysis of some Juristic Techniques for Handling Systematic Defects in the Law", en Bustamante, Thomas y Dahlman, Christian (ed.), Argument Types and Fallacies in Legal Argumentation, Dordrecht, Springer, 2015, p. 157. Cabe precisar que las normas derivadas nos permiten identificar el alcance de las normas. Siguiendo a Alchourrón y Bulygin: «cuando una persona ordena algo, ordena también todas las consecuencias de la proposición que ha ordenado expre- 


\section{VÍCTOR GARCÍA YZAGUIRRE}

por aceptar el refuerzo del antecedente. De acuerdo con este si una proposición p se sigue la proposición q, entonces esta inferencia se mantiene, aunque se añadan nuevas condiciones al antecedente del condicional. En otras palabras, las consecuencias se siguen de las premisas, a pesar de que se incorporen otras premisas al razonamiento. ${ }^{9}$ Si la condición p es suficiente para q, entonces cada vez que tengamos $p$, se seguirá $q$, incluso en casos cuando $p$ concurra con otras condiciones (digamos (p.r)).

\section{B. Norma implícita}

Las normas implícitas son normas que han sido creadas por una autoridad competente en sede de aplicación del derecho. En otros términos, este tipo de normas son la conclusión de haber empleado una técnica de construcción jurídica. ${ }^{10}$

Como podemos ver, la diferencia entre una norma expresa y una norma implícita se basa en si la norma es producto de un acto de interpretación o de construcción. ${ }^{11}$ Por interpretación jurídica se hace referencia a todas aquellas operaciones de atribución de significado a textos normativos. En atención a ello, engloba a todas aquellos métodos y resultados de estos métodos de atribución de significado a textos normativos. En cambio, por construcción jurídica se

samente (aun cuando no esté consciente de ellas). Por ejemplo, si un maestro de escuela ordena que todos sus alumnos deben abandonar el aula, ordena implícitamente que Juan (que es un alumno) debe abandonar el aula, aun cuando no se haya dado cuenta que Juan estaba allí». Alchourrón, Carlos, “Conflictos entre normas y revisión de sistemas normativos”, en Carlos Alchourrón y Eugenio Bulygin, Análisis lógico y derecho, Madrid, Centro de Estudios Constitucionales, 1991, p. 130.

9 Rodríguez, Jorge, "Normas y Razones: aspectos lógicos y sustantivos", Revista Discusiones, núm. 5, 2005, p. 16. La representación formal de la ley del refuerzo del antecedente es (a->b) ->((a.c)->b). Si hay un enunciado x que puede ser inferido de un conjunto de enunciados $y$, entonces x también se infiere de cualquier conjunto de enunciados que sea una extensión de y. La adición de premisas no cancela o invalida la inferencia. En este sentido, si tenemos p->q, será el caso que p.r->q, por ejemplo.

10 Guastini, Riccardo, op. cit., p. 166.

11 Ibidem, p. 49. 
hace referencia a todas aquellas operaciones de creación de normas por parte de los aplicadores del derecho. En este sentido, engloba a todos aquellos métodos y resultados de estos métodos de creación normativa por parte de los aplicadores del derecho.

Una norma implícita, en este sentido, son normas que no han sido formuladas por una autoridad normativa, esto es: i) no se pueden atribuir como un significado posible de un texto normativo, y ii) no pueden ser implicaciones lógicas de un significado posible de un texto normativo. En relación a este último punto, de acuerdo con esta aproximación, las normas obtenidas como resultado de un razonamiento lógico-deductivo (las normas derivadas) no son normas implícitas, pues son el resultado de haber averiguado todas las consecuencias posibles de un determinado significado. En este sentido, no es un acto de creación normativa, sino de haber realizado una operación cognitiva. ${ }^{12}$

Por último, cabe precisar que podemos formular normas derivadas de normas explícitas o de normas implícitas, de forma que tendremos normas derivadas de norma explícita y normas derivadas de normas implícitas. El trabajo de realizar e identificar el conjunto de consecuencias lógicas posibles de las normas es un trabajo que nos permite explicitar los alcances conceptuales de cada una de estas.

En atención a estas precisiones, los conflictos normativos pueden ser, por lo menos, entre: $i$ ) dos normas derivadas de normas expresas; ii) una norma derivada de norma expresa y una norma derivada de norma implícita, o iii) dos normas derivadas de normas implícitas.

\section{Uso de nociones de la lógica deóntica}

Es importante poner de relieve que las discusiones sobre qué son los conflictos normativos son dependientes del uso de la lógica deóntica en el estudio de los sistemas jurídicos. En efecto, la justificación de cómo dos normas están en conflicto entre sí requiere emplear principios lógicos (en específico, el principio de no contradicción), 


\section{para analizar las relaciones entre normas. ${ }^{13}$ Para los fines de este ar- tículo asumiré que la lógica deóntica monotónica es suficiente para}

13 Cabe poner de relieve la precisión formulada por Ratti y Rodríguez quienes bien exponen que en el estudio entre la lógica y el derecho podemos caer en un dilema. Al respecto, si consideramos que la lógica se aplica a normas jurídicas, entonces tenemos que aceptar los principios fundamentales de la lógica en el sistema jurídico (identidad, no contradicción y tercero excluido) lo cual puede ser entendido que esto implica que los sistemas jurídicos son, conceptualmente, consistentes y completos, lo cual es empíricamente falso. En este sentido, o bien aceptamos que la lógica se aplica a las normas, de lo que se sigue que los sistemas son necesariamente consistentes y completos (posición adoptada por los llamados «creyentes»), o bien rechazamos que la lógica se aplica a las normas, lo cual nos lleva a descartar que la lógica sea útil para analizar los sistemas normativos (posición adoptada por los llamados «escépticos»). Al respecto, es posible rechazar el dilema empleando dos tipos de estrategias: i) modificando nuestra forma de entender la lógica deóntica; o ii) diferenciando los tipos de análisis que nos permite hacer la lógica respecto del derecho. Quienes adoptan el camino i), consideran que la lógica debe ajustarse a las características del derecho, de forma tal que se deben formular sistemas lógicos ad hoc (una lógica que admita lagunas, una lógica borrosa, una lógica paraconsistente, una lógica derrotable, entre otras posibilidades). En cambio, quienes adoptan el camino ii) (como son Ratti y Rodríguez, con quienes estoy de acuerdo en este punto), sostienen que aceptar la posibilidad de relaciones lógicas entre normas no nos lleva a que los sistemas jurídicos necesariamente sean consistentes y completos, pues los principios lógicos no son informativos respecto de cómo son (conceptualmente) los sistemas normativos, sino que nos permiten identificar características de estos (si son o no consistentes y completos, en un plano descriptivo). Es decir, son nociones que nos permiten hacer descripciones respecto a cómo son las relaciones lógicas entre normas de un determinado sistema normativo. En efecto, aplicar el principio de contradicción y tercero excluido a las relaciones entre normas no nos lleva a renunciar a la posibilidad de que existan normas inconsistentes ni a que el sistema pueda tener lagunas. Cabe agregar que incluso quienes rechazan la posibilidad de identificar relaciones lógicas entre normas no dejan de emplear estudios de lógica en el derecho, pues las nociones de inconsistencia y completitud son nociones lógicas, lo que es decir que emplean la lógica para dar cuenta de cómo son los sistemas jurídicos. Sobre esta discusión y con otras precisiones relevantes ver Ratti, Giovanni Battista y Rodríguez, Jorge, "Los principios fundamentales de la lógica en el derecho", Analisi e Diritto, 2018-1 y Rodríguez, "Contradicciones normativas: jaque a la concepción deductivista de los sistemas jurídicos", Doxa, Vol. 17-18, 1995, pp. 363 y ss. 
APUNTES CONCEPTUALES PARA LA IDENTIFICACIÓN...

poder dar cuenta de los conflictos normativos (podemos emplearla para describir que un sistema normativo es inconsistente). ${ }^{14}$

De acuerdo con el principio de no contradicción, no puede ser el caso que una cierta proposición p sea, a la vez, verdadera y falsa. Ello, en un análisis normativo (analizamos proposiciones modalizadas con un operador deóntico de forma Op, por ejemplo), refiere a que no puede ser el caso que una cierta norma y su negación sean ambas válidas (entendido como aplicables) para un mismo caso. En otros términos, dos normas son inconsistentes entre sí cada vez que no se satisfaga el principio de no contradicción, ${ }^{15}$ de acuerdo con el cual, no puede ser el caso una norma y su negación sean ambas aplicables a un mismo caso conforme al sistema normativo. ${ }^{16}$

Uno de los principales problemas de la inconsistencia es que genera la frustración de una de las funciones de las normas jurídicas:

14 Sobre este punto ver Ratti, Giovanni Battista, El Gobierno de las normas, Madrid, Marcial Pons, 2013, pp. 196 y ss.

15 Representado formalmente como $\neg(\mathrm{p} . \neg \mathrm{p})$ para proposiciones y como $\neg(0 p . \neg 0 p)$ para normas. El principio de no contradicción puede ser entendido de dos maneras. Si es entendido de forma prescriptiva, entonces lo que estamos diciendo es que el creador del derecho no debe crear normas que entren en conflicto con otras. Si es entendido de forma descriptiva, entonces lo que estamos diciendo es que, conforme al principio lógico de no contradicción, un sistema es o bien consistente (no presenta inconsistencias) o bien no es consistente (presenta consistencias), sin que ello implique un deber ser o excluyendo alguna posibilidad descriptiva. En mi reconstrucción de esta discusión estoy empleando un uso descriptivo del principio de no contradicción.

16 Ratti, Giovanni Battista y Rodríguez, Jorge, "Los principios fundamentales de la lógica en el derecho”, Analisi e Diritto, 2018-1, p. 161. Debo precisar que adopto la definición de estos autores, pues también asumo que las normas, al ser entidades prescriptivas, carecen de valores de verdad. A efectos de poder analizar las relaciones lógicas entre normas, se sustituye la noción de verdad por la de validez. Esto nos lleva al problema de cómo entender la validez, pues es un concepto ambiguo (puede ser entendido como pertenencia, fuerza obligatoria, aplicable, existencia formal o eficacia entendida como empleada para resolver el caso). En este punto, he elegido el sentido de aplicabilidad para efectos de precisión con la idea que estoy tratando de expresar (no podría ser, por ejemplo, pertenencia, validez entendida como pertenencia, pues la consistencia no es un rasgo necesario de los sistemas jurídicos). En las líneas siguientes seré expreso en señalar qué sentido de validez empleo para dar cuenta de cada concepción de conflicto normativo analizada. 


\section{VÍCTOR GARCÍA YZAGUIRRE}

poder guiar la conducta de sus destinatarios. En lógica proposicional se da cuenta de las situaciones de inconsistencia a través del principio ex falso sequitur quodlibet, de acuerdo con el cual, ante un caso de contradicción se sigue cualquier conclusión. ${ }^{17}$ En este sentido, un conflicto normativo refiere a una situación en la que no es posible la aplicación conjunta de dos normas, pues de hacerlo, cualquier resultado normativo sería posible. En este punto es pertinente precisar que los juristas tenemos una forma diferente de entender los conflictos normativos a cómo la tienen los lógicos (aunque ambos empleamos la lógica deóntica para analizar las relaciones entre normas). Para los juristas, como vemos, un conflicto es entendido como la conjunción de dos normas condicionales: (p->0q) y (p->0 ᄀq) ${ }^{18}$

Como bien nos aclara Giovanni Battista Ratti, en la lógica proposicional la conjunción de p->0q y p->0 ᄀq equivale a la negación del antecedente, es decir, $\neg$ p, de forma que la relación entre estas dos normas no es inconsistente (contrario a lo que pensamos los juristas, este no es un caso de conflicto). ${ }^{19}$ Por ejemplo, si considera-

17 Sobre este punto ver Rodríguez, Jorge, "Contradicciones normativas: jaque a la concepción deductivista de los sistemas jurídicos", Doxa, vol. 17-18, 2005, p. 358. Para analizar este mismo problema desde una concepción expresiva de las normas, véase Alchourrón, Carlos, "Conflictos entre normas y revisión de sistemas normativos", cit., p. 141.

18 Debo precisar que para efectos explicativos estoy dando cuenta de la noción de conflictos normativos empleando normas condicionales bajo la concepción puente. Sin perjuicio de ello, estas consideraciones también son aplicables a normas categóricas ( $\mathrm{pp}$ y 0$\urcorner p$, por ejemplo) y a normas condicionales bajo la concepción insular $(\mathrm{O}(\mathrm{p}->\mathrm{q})$ y $\mathrm{O}(\mathrm{p}->\neg \mathrm{q})$, por ejemplo).

19 Ratti, Giovanni Battista, "Negation in legislation", en Araszkiewicz, Michał y Płeszka, Krysztof (ed.), Logic in the Theory and Practice of Lawmaking, Cham, Springer, pp. 137-158. 143. El caso es el siguiente: tenemos un sistema normativo com-

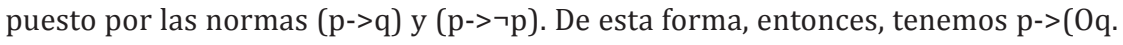
$0\urcorner q)$. Si a este premisa le agregamos $\neg(0 q .0\urcorner q)$ el resultado es una inferencia válida: $\neg$ p. En atención a ello, este no es un caso de ex falso sequitur quodlibet, pues lógicamente sí es posible realizar deducciones válidas.

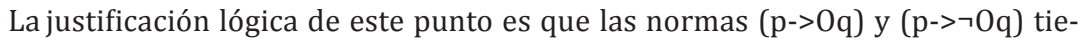
nen una relación (conforme al cuadro de oposición deóntica) de subcontrariedad, es decir, ambos pueden ser aplicables (o verdaderos si estuviéramos en lógica proposicional), pero no pueden ser ambos no aplicables (o falsos). En caso por lo menos uno de ellos no sea aplicable es el caso que el otro, necesariamente, es aplicable. 
APUNTES CONCEPTUALES PARA LA IDENTIFICACIÓN...

mos conjunto de normas "Juan es ladrón, entonces obligatorio castigarlo", con "Juan es ladrón, entonces obligatorio no castigarlo" podemos derivar lógicamente $\neg$ p, es decir, "Juan no es un ladrón" ${ }^{20}$

Esto supone una diferencia disciplinar entre cómo los lógicos deónticos y los juristas que emplean la lógica deóntica para analizar los sistemas jurídicos. ${ }^{21}$ Sin perjuicio de ello, los juristas dependemos de los análisis lógicos para poder hablar de conflictos en el derecho, en específico, de entender y aplicar el refuerzo del antecedente.

El refuerzo del antecedente nos permite señalar que cada vez que se verifique una propiedad, entonces se sigue el consecuente normativo. Si esto es así, entonces cada vez que verifiquemos que una propiedad posee dos calificaciones normativas incompatibles entre sí tendremos un caso de conflicto normativo. En efecto, si dejamos de lado el refuerzo del antecedente no podemos determinar el contenido de los antecedentes ni mucho menos si dos antecedentes están conectados a consecuencias normativas incompatibles entre sí.

El refuerzo del antecedente aplicado a los antecedentes de las normas nos permite explicar cómo dos normas pueden entrar en conflicto entre sí. En efecto, si tenemos una norma p->0q, sabemos que se aplica dicha norma a pesar de que concurra con las propiedades $r, z$, o cualquier otra. Bajo esta premisa si tenemos un conjunto compuesto por p->0q y r->0ᄀq, por refuerzo del antecedente, sabemos que hay un conflicto entre ambas, pues en caso de (p.r)

Pero, a la inversa, si uno de ellos es aplicable, no es necesariamente el caso que el otro lo sea. Ratti, Giovanni Battista y Rodríguez, Jorge, “On Coherence as a Formal...”, cit., p. 135. Ratti, Giovanni Battista, "Rational Law-Giving: Structural and Interpretative Queries", Analisi e Diritto, 2017, pp. 86-88.

20 Sobre este punto, Hage, Jaap, "Rule Consistency", Law and Philosophy, num. 19, 2000, p. 371.

21 Este punto ha sido señalado por Ratti quien sostiene que los juristas empleamos un aparato lógico precario en comparación al que disponen los lógicos deónticos. Con mayor detalle, la racionalidad lógica (poder hacer deducciones correctas) no es la misma que la racionalidad jurídica, como queda mostrado en el caso de cómo se entienden las inconsistencias. Ratti, Giovanni Battista, "Rational law-giving...", cit., p. 88. 
serán aplicables dos consecuencias normativas lógicamente incompatibles entre sí: 0 p y $0 \neg q .{ }^{22}$ Sobre este punto volveré más adelante.

\section{Conflicto normativo como imposibilidad de cumplimiento conjunto por el destinatario}

Los conflictos normativos suelen ser entendidos por los juristas como aquellos casos en los cuales el destinatario de las normas no puede cumplir dos o más obligaciones jurídicas aplicables en un mismo tiempo-espacio. ${ }^{23} \mathrm{Al}$ respecto, esta imposibilidad de cumplimiento puede ser generada por razones lógicas o por razones empíricas.

\section{A. Imposibilidad lógica}

La imposibilidad lógica de cumplimiento se genera debido a que, por lo menos, dos normas regulan el mismo caso genérico, pero con consecuencias normativas diferentes e incompatibles entre sí. ${ }^{24} \mathrm{Di}$ cho de forma más precisa, tenemos un conflicto cada vez que tengamos un caso de modalización deóntica inconsistente respecto a dos

22 Ratti, Giovanni Battista, "Normative Inconsistency and Logical Theories: a First Critique of Defeasibilism", en Araszkiewicz, Michał y Šavelka, Jaromir (ed,), Coherence: Insight from Philosophy, Jurisprudence and Artificial Intelligence, Dordrecht, Springer, 2013, p. 126.

${ }^{23}$ Debo reiterar que en este artículo sólo voy a dar cuenta de conflictos normativos entre normas, dejaré de lado los conflictos normativos entre proposiciones normativas. En efecto, una lógica de normas y una lógica de proposiciones no son intensional ni extensionalmente equivalentes. Para ver este punto Navarro, Pablo y Rodríguez, Jorge, "Entailed Norms and the Systematization of Law", en Araszkiewicz, Michał y Płeszka, Krysztof (ed.), Logic in the Theory and Practice of Lawmaking, Cham, Springer, 2015, p. 101.

24 Debo precisar que esto presupone que estas normas son aplicables, es decir, hay una tercera norma que establece que estas dos normas en conflicto deben ser empleadas por el aplicador del derecho para resolver el caso individual. 
APUNTES CONCEPTUALES PARA LA IDENTIFICACIÓN...

casos genéricos total o parcialmente idénticos, ${ }^{25}$ lo cual implica un problema de inconsistencia entre estas dos normas. ${ }^{26}$

Esta definición ha sido sostenida por diversos autores. ${ }^{27} \mathrm{Ha}$ sido formulada desde el punto de vista del destinatario de las normas (prescriptivas) y se centra en el análisis de consistencia entre dos o más normas aplicables a un mismo caso. En efecto, bajo esta propuesta tenemos un caso de conflicto normativo cada vez que le sea imposible al destinatario cumplir el mandato de dos normas prescriptivas, pues el cumplimiento conlleva incumplir la otra. ${ }^{28}$

25 Cabe precisar, siguiendo a Hage, que la inconsistencia entre normas es independiente de si los hechos que satisfacen los antecedentes de las normas ocurren o no. En este sentido, la inconsistencia depende de: i) la incompatibilidad de los consecuentes; y de ii) la compatibilidad de las condiciones previstas en el antecedente. Hage, Jaap, "Rule Consistency", cit., p. 372.

26 Martínez Zorrilla, con acierto, hace una distinción interesante partiendo de este punto. A partir de la idea de que el derecho tiene como función guiar el comportamiento de sus destinatarios, la noción de conflicto normativo nos permite poner de relieve todos aquellos casos en los cuales esto no se logra por prever pautas de comportamiento incompatibles. Este problema puede ser entendido de dos formas: i) como un defecto formal del sistema normativo, o ii) como un problema de justificación (toda solución es viable, pues todas estarían justificadas). De acuerdo con i) el problema de la justificación de los comportamientos es un efecto derivado de tener un sistema lógicamente defectuoso. Martinez Zorrilla, David, "Conflictos normativos", en Fabra Zamora, Jorge y Nuñez Vaquero, Álvaro (ed.), Enciclopedia de filosofía y teoría del derecho, México, UNAM, 2015, vol. 2, pp. 1307-09. En lo que sigue de este apartado asumiré la aproximación $i$ ), pues es la que usualmente emplean los juristas (la aproximación ii) suele ser empleada por los teóricos morales para aclarar la noción de dilema moral). Asimismo, dado que una justificación sólo es racionalmente controlable si esta es inferida de una premisa normativa (de una norma), de forma que la distinción entre defectos lógicos del sistema y problemas de justificación es útil para esclarecer aspectos distintos de una misma dificultad, pero no para dar cuenta de problemas independientes.

27 Por todos, véase Ross, Alf, Sobre el derecho y la justicia, Buenos Aires, Eudeba, 1963 p. 124, y Gavazzi, Gioacomo, Delle antinomie, Giappichelli, Turín, 1959, pp. 52-55.

28 Navarro, Pablo y Rodríguez, Jorge, Deontic Logic and Legal Systems, Cambridge, Cambridge University Press, 2014, p. 176. En términos de Eugenio Bulygin: “[1] a presencia de soluciones contradictorias lleva necesariamente a la desobediencia al derecho: siendo incompatibles las dos soluciones, al menos una de ellas tendrá que ser dejada de lado y la norma que la establece será desobedecida". Bulygin, 


\section{VÍCTOR GARCÍA YZAGUIRRE}

A efectos de precisión, siguiendo a Chiassoni, estaremos en un caso de conflicto normativo cada vez que un sujeto sea el destinatario de dos normas prescriptivas que establecen consecuencias lógicamente incompatibles para un mismo supuesto de hecho. Esto implica una imposibilidad de seguir simultáneamente estas dos normas en relación a la acción o estado de cosas previsto de verificarse el caso genérico. ${ }^{29}$

Siguiendo la terminología (y concepción del derecho como un sistema normativo ordenado) de Alchourrón y Bulygin, podemos entender que un conflicto normativo refiere a que un caso del Universo de Casos está correlacionado con dos o más soluciones incompatibles del Universo de Soluciones. ${ }^{30}$

Esta noción, como podemos ver, presupone tres requisitos formales para la generación de un conflicto normativo: coincidencia en el caso genérico regulado, aceptar el refuerzo del antecedente y soluciones normativas incompatibles entre sí.

En relación a la aplicabilidad de las normas a un mismo caso (un caso individual se subsume en el caso genérico de dos normas), refiero que las normas deben tener el mismo ámbito de aplicación (se apliquen en un mismo momento, a un mismo lugar, a los mismos sujetos y regule una misma acción o estado de cosas). ${ }^{31}$ Cabe ano-

Eugenio, “Teoría y técnica de la legislación”, Análisis lógico y derecho, Madrid, CEC, 1991, p. 418.

29 Chiassoni, Pierluigui, Técnicas de interpretación jurídica, breviario para juristas, Madrid, Marcial Pons, 2011, p. 291. En un sentido similar, Silvia Zorzetto aclara que, desde un plano lógico, los conflictos son generados cada vez que dos normas prescriben cosas incompatibles. Situación que no es contingente, no es fáctica (no es una cuestión empírica dependiente de las acciones), no concierne a hechos, sino a los conceptos contenidos en las normas: es un problema de tener modalidades de calificación deónticas incompatibles entre sí aplicables al caso. Zorzetto, Silvia, La norma speciale. Una nozione ingannevole, Pisa, ETS, 2010, p. 516.

30 Alchourrón, Carlos y Bulygin, Eugenio, Sistemas normativos, Introducción a la metodología de las ciencias jurídicas, 2a. ed., Buenos Aires, Astrea, 2012, pp. 92 y ss.

31 Iturralde, Victoria, "Una aproximación al tema de las antinomias normativas: problemas relativos a la identificación de las mismas", Anuario de filosofía del derecho, núm. 4, 1987, p. 333. Cabe agregar una precisión. Supongamos que tenemos un sistema normativo compuesto por dos normas: p->0q y $\neg$ p->0q. En este sistema podemos inferir que tenemos la norma (p.าp)->0q, la cual tiene una condición de 
APUNTES CONCEPTUALES PARA LA IDENTIFICACIÓN...

tar que dos normas que no regulan el mismo supuesto de hecho, en abstracto, sí pueden entrar en conflicto entre sí como un conflicto de instanciación, pero sobre estos me ocuparé en el siguiente subapartado.

En cuanto a "consecuencias lógicamente incompatibles", debo precisar que ello tiene dos sentidos posibles: que el destinatario tenga que cumplir normas que son contradictorias o contrarias entre sí. Profundizar este punto nos llevaría a especificar todas las posibilidades de cómo entender las relaciones lógicas entre normas. ${ }^{32}$ Mi propósito, en este momento, es más modesto: me interesa poder poner de relieve la distinción conceptual entre estos dos posibles casos de inconsistencia, pues cada uno de estos conlleva métodos de solución distintos. En atención a ello, asumiré la propuesta de que las normas no tienen valor de verdad y consideraré que el valor lógico de las normas (para poder dar cuenta de las relaciones lógicas entre estas) recae en su validez entendida como eficacia. ${ }^{33}$ En este sentido:

aplicación imposible, de forma que no podríamos hablar de contradicción o conflicto normativo en caso dos normas tengan antecedentes que se contradicen entre sí. Sobre el punto general y la precisión agregada, Ratti, Giovanni Battista, "Normas jurídicas y contradicciones", en Redondo, María Cristina y Navarro, Pablo (eds.), La filosofía desde el derecho, homenaje a Ricardo Caracciolo, México, Fontamara, 2016, pp. 243-236.

32 Aclarar este punto, además, nos llevaría a dar cuenta de todas las formas de solucionar el dilema de Jørgensen. Al respecto, en la literatura especializada nos podemos encontrar con, por lo menos, estas propuestas de solución:

Considerar que las normas poseen valores de verdad (asumiendo, por ejemplo, una concepción semántica de mundos deónticos posibles), por lo que es posible una lógica de normas.

i. Considerar que las normas carecen de valores de verdad, por lo que no es posible una lógica de normas.

ii. Considerar que las normas carecen de valor de verdad, pero podemos analizar las relaciones lógicas entre normas reemplazando la noción de verdad por satisfacción, fuerza obligatoria o pertenencia para formular una lógica de normas.

iii. Considerar que las normas carecen de valores de verdad, por lo que debemos formular una lógica de normas que no emplee la noción de verdad (por ejemplo, empleando una noción abstracta de consecuencia).

Sobre estas posibilidades ver Navarro, Pablo y Rodríguez, Jorge, Deontic logic..., cit., cap. II.

33 Para este punto sigo a Ratti, Giovanni Battista, "Negation in legislation", cit., 


\section{VÍCTOR GARCÍA YZAGUIRRE}

i) Dos normas son conjuntamente contrarias si ambas pueden ser inefectivas, pero no ambas pueden ser efectivas. Por ejemplo, pensemos en un conjunto compuesto por (p->0q) y (p->0 ᄀp).

ii) Dos normas son conjuntamente contradictorias en aquellos casos en los cuales, si una norma es eficaz, entonces la otra no lo puede ser. Por ejemplo, pensemos en un conjunto compuesto por $(\mathrm{p}->\mathrm{Oq})$ y $(\mathrm{p}->\mathrm{F} \neg \mathrm{q}){ }^{34}$

En ambos casos el destinatario al cumplir una de las normas generará, necesariamente, el no cumplimiento de la otra. Este punto, además, permite poner de relieve que los conflictos normativos generados a partir de la modalización deóntica y no (únicamente) por el contenido de la norma. En efecto, consideremos que tenemos la acción p y su caso complementario $\neg$ p. Si bien ambas son incompatibles entre sí, cabe anotar que entran en conflicto a partir de cómo están calificadas deónticamente y no por el contenido: si tuviéramos en el caso de Pp y $\mathrm{P} \neg$ p, bajo esta forma de entender los conflictos, no habría un conflicto normativo, pues la realización del permiso de una acción no conlleva al no cumplimiento del permiso para no realizar la acción. En este sentido, como bien apunta Navarro y Rodríguez, la contradicción entre los contenidos normativos es una condición necesaria pero no suficiente para caracterizar las contradicciones normativas. ${ }^{35}$

p. 140. Se emplea la noción de eficacia a efectos de atender la función del derecho de guiar la conducta: solo siendo eficaz, es decir, siendo empleada como premisa normativa, se satisface esta finalidad. En caso de normas incompatibles entre sí, una implica la ineficacia de la otra.

34 Esta diferencia, siguiendo a Ratti, también puede ser explicada a través de la noción de negación interna y externa. Veamos:

i. Incompatibilidad lógica por contradicción: refiere al conflicto entre una norma y su negación externa. En términos formales alude a que $0 p$ es contradictoria con $\neg 0$ p (que equivale a $\mathrm{P} \neg \mathrm{p}$ ). A la inversa, $\mathrm{O} \neg \mathrm{p}$ (que equivale a $\mathrm{Php}$ ) es contradictoria con Pp.

ii. Incompatibilidad lógica por contrariedad: refiere al conflicto entre una norma y su negación interna. En términos formales alude a que 0 p es contraria a 0$\urcorner p$. A la inversa, 0$\urcorner$ p (equivalente a Php) es contraria a 0 p.

Véase Ratti, Giovanni Battista, "Normas jurídicas y contradicciones”, cit., p. 233.

35 Navarro, Pablo y Rodríguez, Jorge, Deontic logic..., cit., p. 177. 
APUNTES CONCEPTUALES PARA LA IDENTIFICACIÓN...

\section{B. Imposibilidad empírica}

La imposibilidad empírica de cumplir con prescripciones jurídicas es un problema inicialmente planteado por Hilpinen. En adelante llamaré a estos supuestos como conflictos de instanciaciones y refiere a los casos en los cuales, por razones fácticas, no es posible cumplir con todas las consecuencias lógicas derivables de las normas del sistema aplicables a un sujeto en un determinado momento. En otros términos, da cuenta de los casos en los que un destinatario no puede cumplir con todas las instanciaciones de una misma o diferentes normas, pues cumplir con una de ellas implicaría incumplir con otra norma derivada.

Por instanciación refiero a los supuestos por los cuales podemos considerar que un determinado objeto (abstracto o empíricamente verificable), es un ejemplo de una propiedad prevista en el antecedente de una norma. Instanciar, en este sentido, refiere a que un determinado objeto es una instancia o ejemplar de una determinada clase de objetos.

Este tipo de conflictos de instanciación da cuenta de dos tipos de casos: en primer lugar, refiere al supuesto que de una norma general podemos derivar dos o más normas individuales (aplicaciones de la norma general a casos individuales, dicho de otra forma, instanciaciones o ejemplificaciones) que pueden generar una situación conflictiva, en tanto, el cumplimiento de una norma individual derivada una determinada norma conlleva al cumplimiento de otra o del resto de normas individuales derivadas de la misma norma general.

Al respecto, Navarro y Rodríguez ofrecen un ejemplo bastante ilustrativo: ${ }^{36}$ imaginemos que en un hospital hay un solo médico el cual se rige por una norma que prescribe "si médico, entonces obligatorio tratar a los pacientes con paro cardiaco de forma inmediata”. Pues ocurrió que el médico fue notificado, en un mismo momento, de tres pacientes que estaban sufriendo de un paro cardiaco (todos en simultáneo). En este caso el médico no puede cumplir con sus obligaciones jurídicas, pues le es físicamente imposible poder dar

36 Ibidem, p. 183. Señalan que el ejemplo fue propuesto originalmente por Hansson y Makinson. 


\section{VÍCTOR GARCÍA YZAGUIRRE}

atención a los tres pacientes a la vez y es el caso que atender a un paciente implica dejar de atender a los otros dos.

En segundo lugar, refiere al supuesto en el que dos normas generales no regulan el mismo caso genérico, pero dadas ciertas circunstancias del caso analizado se produce un supuesto en el que se pueden formular normas derivadas lógicamente incompatibles entre sí. En estos supuestos, la imposibilidad de cumplir por parte del destinatario sólo se genera en determinadas circunstancias fácticas (en otros términos, dos normas no entran en conflicto en todas las circunstancias posibles, sólo en algunas).

Al respecto, Rodríguez ha formulado un ejemplo claro: ${ }^{37}$ imaginemos que nos regimos bajo un sistema normativo que contiene dos normas. La primera, N1, señala que "si conductor de auto, entonces obligatorio detenerse cuando el semáforo de luz roja”, y la segunda, N2, establece que "si conductor de auto, entonces prohibido detenerse frente a una base militar". Estas dos normas, como se puede ver, no entran en conflicto en abstracto, pero podemos tener circunstancias en las cuales tengamos normas derivadas que sí entren en conflicto: el caso que hayan puesto un semáforo frente a una base militar y un conductor tenga que enfrentarse a la decisión respecto si detenerse frente a la luz roja (conforme a N1) o no detenerse frente a la base militar (conforme a N2). ${ }^{38}$

Los conflictos de instanciación refieren a aquellos casos en los cuales dos normas individuales son incompatibles entre sí. Es un tipo de conflicto normativo que no se detecta a través del análisis del sistema normativo (no es un caso de verificar en el sistema el con-

37 Rodríguez, Jorge, Lógica de los sistemas jurídicos, Madrid, CEPC, 2002, p. 9.

38 Sobre este punto cabe precisar que este tipo de conflictos de instanciación son posibles siempre que la propiedad prevista en una norma no implique la negación de la propiedad prevista en otra norma. En este sentido, si tenemos dos normas con antecedentes p y r, respectivamente, no tendremos este tipo de conflictos de instanciación normas derivadas de estas normas si p no implica $\neg$ r o r no implica $\neg$ p. Ratti, Giovanni Battista, "Normas jurídicas y contradicciones", cit., p. 234. Al respecto, considero que este es justamente el problema del primer tipo de conflicto de instanciación, pero sobre este punto precisaré de inmediato. 
APUNTES CONCEPTUALES PARA LA IDENTIFICACIÓN...

junto de (p->0q) y (p->0ᄀp)), sólo puede ser verificado una vez que hemos considerado los hechos de un caso individual. ${ }^{39}$

El punto que me interesa poner de relieve en este momento es si, conceptualmente, los conflictos de instanciación (generados por imposibilidad fáctica) están dando cuenta de un problema diferente a los conflictos normativos generados por imposibilidad lógica. Al respecto, conforme lo señalado al inicio de este apartado, considero que no, pues estos supuestos reformulados de un mismo problema: incompatibilidad lógica de dos normas. Pero dado que tenemos dos tipos de casos esto requiere formular dos tipos de precisiones respecto de cada uno.

En relación con el supuesto de conflicto entre normas derivadas de diferentes normas generales, retomemos el ejemplo del semáforo en luz roja y la base militar. En este supuesto tendríamos dos normas que no entran en conflicto normativo: N1 está compuesta por (p->0q) y N2 está compuesta por $(\mathrm{r}->0 \neg q)$. El conflicto de instanciación se genera si tenemos un caso compuesto por las propiedades (p.r), es decir, un semáforo indicando luz roja frente a una base militar.

Como bien anota Giovanni Battista Ratti, considerar que este es un sentido de conflicto normativo sería confundir la pregunta por la ocurrencia empírica en la que surge una antinomia con la pregunta por su identificación conceptual. ¿Qué justifica que en el caso de (p.r) tengamos un conflicto normativo? Lo justifica el mismo criterio empleado en el subapartado anterior: estamos frente a un supuesto de imposibilidad lógica entre ((p.r)->0q) y (r.p)->0 ᄀq).

En efecto, que sean los hechos del caso aquellos que sustenten determinados tipos de inferencias a partir de ciertas normas aplicables no justifica que estemos frente a un conflicto normativo. Eso sólo daría cuenta del carácter contingente de este tipo de situaciones o, dicho con más precisión, de su posibilidad empírica. La justificación de porqué son conflictos es que se genera una imposibilidad

39 En términos de Alchourrón, este tipo de conflicto da cuenta que (en caso de tener normas condicionales): "[u]n conjunto de normas puede ser inconsistente vía determinados hechos y consistente vía otros hechos". Alchourrón, Carlos, "Conflictos entre normas y revisión de sistemas normativos", cit., p. 300. 


\section{VÍCTOR GARCÍA YZAGUIRRE}

lógica entre ellos que conlleva al imposible cumplimiento de dos deberes jurídicos. ${ }^{40}$

En relación al supuesto de conflicto entre normas derivadas de una misma norma general. Retomemos el ejemplo del médico que tenía la obligación atender a varios pacientes (asumamos que son dos) con paro cardiaco a la vez, pero al atender a un paciente ello implica desatender al otro (y por ende incumplir con su obligación). En este caso tendríamos diferentes normas individuales derivadas de una misma norma general las cuales no pueden ser cumplidas, pues el cumplimiento de una supone el incumplimiento de las otras.

Esto nos lleva a un punto similar al anterior. ¿Esto nos permite justificar por qué las normas individuales están en conflicto normativo entre sí? No, sólo nos da cuenta de un supuesto de imposibilidad material para satisfacer todas las normas derivadas. Lo que nos permite este caso es mostrar que estamos frente a un supuesto de incompatibilidad conceptual (lo cual excluye la posibilidad de considerar que estamos frente a un conflicto normativo). ${ }^{41}$

Conforme al ejemplo estamos ante un supuesto de imposibilidad material para cumplir con todas las normas derivadas de la norma general, lo que quiere decir que realizar una determinada acción implica, conceptualmente, no hacer otras acciones. A efectos de representación, demos cuenta de cada paciente mediante un símbolo: el primer paciente como p1 y el segundo paciente como $\mathrm{p} 2$. Si no es posible atender a p1 y p2 al mismo tiempo, ello quiere decir que atender a un paciente implica no atender al otro. Esto lo podemos representar como «si p1, entonces ᄀp2»y «si p2, entonces ᄀp1».

Si esto es correcto, entonces de la norma general tendríamos dos normas derivadas distintas:

Norma derivada 1: si médico, entonces obligatorio atender a p1 (el cual implica $\neg$ p2)

Norma derivada 2: si médico, entonces obligatorio atender a p2 (el cual implica $\neg \mathrm{p} 1$ )

40 En un sentido similar Orunesu, Claudina, Positivismo jurídico y sistemas constitucionales, Madrid, Marcial Pons, 2012, p. 82.

41 Agradezco a Giovanni Battista Ratti por ayudarme a esclarecer este punto. 
APUNTES CONCEPTUALES PARA LA IDENTIFICACIÓN...

Como podemos ver, no tenemos consecuencias lógicas incompatibles entre sí, pues cada una de estas supone, conceptualmente, obligaciones diferentes. En este sentido, no habría conflicto normativo.

\section{Conflicto normativo como actos irracionales o anormales del legislador}

Para algunos autores dos normas conflictúan entre sí a causa de la discrepancia entre lo que pretende prescribir un legislador racional y los términos empleados para ello. Esta propuesta fue formulada como una manera alternativa para enfrentar el problema de cómo dar cuenta de este tipo de defectos lógicos en el sistema sin renunciar a la idea de que las normas carecen de valores de verdad.

Alchourrón y Bulygin; ${ }^{42}$ a diferencia de la concepción anterior, propusieron no enfocarse en el destinatario de las normas, sino en el legislador. De acuerdo con estos autores, si tenemos un conjunto normativo compuesto por 0 p y 0$\urcorner$ p podemos decir que estas son incompatibles entre sí porque son incompatibles con las intenciones de la autoridad normativa. La incompatibilidad se genera debido a que una orden del legislador no podría ser realizada de manera conjunta con otra orden del mismo legislador. La inconsistencia, en este sentido, revela la no coincidencia entre las intenciones del creador de la norma y las expresiones verbales empleadas. ${ }^{43}$ En efecto, normalmente un legislador emite órdenes con la intención de que sean cumplidas, lo cual implica que aquello que ordene no implique, a la vez, desobedecerle.

Asumiendo un enfoque y preocupación similar, Von Wright (partiendo de la premisa que las normas carecen de valor de verdad y

42 Alchourrón y Bulygin, Fundamentos pragmáticos para una lógica de normas. Analisis lógico y derecho, Madrid, CEC, 1991, pp. 160 y ss.

43 Ibidem, p. 161. En un sentido similar Alf Ross señaló: “[1]a contradicción (incompatibilidad) tiene lugar cuando dos directivos reflejan una inconsistencia (irracionalidad) en la voluntad de la autoridad que manda, en el sentido de que esta quiere acciones incompatibles". Ross, Alf, Lógica de normas, Madrid, Tecnos, 1971, p. 158. 
preocupado por analizar si Op y 0$\urcorner$ p son contradictorias), ${ }^{44}$ propuso una conceptualización similar a la de los profesores argentinos. Para este autor, hemos de considerar la racionalidad del legislador que emite las órdenes: un legislador que exige que el mismo estado de cosas sea y no sea el caso no puede tener sus exigencias satisfechas. Como vemos, Von Wright emplea una terminología propia, pero está dando cuenta del mismo concepto que Alchourrón y Bulygin. El autor finlandés considera que los conflictos normativos dan cuenta de actos de irracionalidad del legislador, esto es, de ordenar a un destinatario a realizar y no realizar actos a la vez.

Esta forma de entender los conflictos normativos traslada la discusión de consistencia e inconsistencia al nivel de condiciones normales y anormales o de racionalidad e irracionalidad. Este discurso es una manifestación de entender que el legislador como agente racional, lo cual es una caracterización ideal de que los creadores de normas que asume que estos pretenden crear sistemas normativos consistentes y completos desde un punto de vista lógico.

La discusión sobre el legislador racional es amplísima y muy diversa, por lo que no es oportuno poder profundizar sobre este punto. Sin perjuicio de ello, este punto me permite retomar una distinción introducida anteriormente. Esta forma de presentar la racionalidad es problemática, pues nos puede llevar a resultados que sean contraintuivos para los juristas. Si entendemos la racionalidad del legislador de manera que este se debe guiar por los principios de la lógica (de forma que sus productos legislativos deberían ser consistentes y completos), entonces el conjunto de normas (p->0q) y ( $\mathrm{p}->0\urcorner \mathrm{p})$ no nos permite dar cuenta de un conflicto normativo. Esto, como ya lo había señalado al inicio de este apartado, debido a que de

44 Von Wright, "Is there a Logic of Norms?", Ratio Juris, vol. 4, núm. 3, 1991, p. 271. Este autor está pensando en describir la lógica de las normas como una forma de describir la racionalidad del legislador (véase, además, Rodríguez, Jorge, "Sistemas normativos, lagunas jurídicas y clausura lógica", Anuario de Filosofía del Derecho, 2015, p. 17). Debo precisar aquí que este enfoque no renuncia ni descarta entender la consistencia como la posibilidad de que el destinatario pueda conjuntamente satisfacer lo que se le exige mediante dos o más normas aplicables al caso. El punto de atención está puesto en porqué se generan estos escenarios de inconsistencia. 
APUNTES CONCEPTUALES PARA LA IDENTIFICACIÓN...

estas normas uno puede inferir, válidamente, $\neg$ p. En este sentido un legislador que califica una misma acción con dos consecuencias lógicas diferentes y que no pueden ser cumplidas a la vez, no está siendo (en un sentido lógico) irracional, pues podemos hacer inferencias de este conjunto de normas. ${ }^{45}$

\section{Clasificación de los conflictos normativos}

La clasificación de los conflictos normativos depende del criterio que empleemos para justificar cómo dos normas pueden entrar en conflicto entre sí, como de la noción de norma que estemos asumiendo. En este apartado daré cuenta una propuesta formulada a partir de la comprensión de los conflictos normativos como casos de imposibilidad lógica de cumplimiento de las prescripciones ordenadas entre, por lo menos, dos normas.

En las líneas siguientes adoptaré la propuesta originalmente ofrecida por Alf Ross atendiendo a su impacto en la teoría del derecho contemporánea y por su rendimiento explicativo. ${ }^{46}$ Bajo este modelo, se emplea como criterio de clasificación todas las posibilidades de relación entre los antecedentes de las normas que están en conflicto (conectan a consecuencias normativas diferentes y lógicamente incompatibles). En este sentido, los conflictos normativos pueden ser:

a) Conflictos total-total entre dos o más normas: en estos casos dos normas comparten el mismo antecedente. ${ }^{47}$ Esto implica que no es posible aplicar una norma sin evitar que entre en conflicto con la otra. Para ser más precisos, en este tipo de ca-

45 Esta crítica es formulada por Ratti, Giovanni Battista, "Rational Law-Giving...", cit., pp. 85 y ss.

46 Ross, Alf, Sobre el derecho y la justicia, cit., pp. 124-126. Para ver otro conjunto de propuestas es muy interesante el texto de Mazzareze, Tecla, "Antinomie, paradossi, logica deontica", Rivista internazionale di filosofia del diritto, vol. 61, núm. 4, 1984.

47 Sólo por razones de presentación asumiré aquí y en el resto de tipos de conflictos normativos que estamos frente a un conflicto entre dos normas, pero esto no implica la imposibilidad de que sea entre más normas. 
sos cada norma regula al mismo sujeto, la misma acción o estado de cosas, en el mismo momento y para el mismo lugar.

Formalmente, esto se representa como el conjunto de estas dos normas: $(p->0 q)$ y $(p->0 \neg q)$. Como podemos ver, ambas normas regulan el mismo caso genérico $\mathrm{p}$, lo cual conlleva a una inevitable situación de inconsistencia.

b) Conflictos total-parcial entre dos o más normas: en estos casos una de las normas tiene un antecedente que es igual a un fragmento del antecedente de otra norma. En otros términos, una de las normas tiene un antecedente compuesto por un conjunto de propiedades que está también previsto por completo por el antecedente de otra norma, pero que, además de estas, prevé propiedades adicionales (uno de los antecedentes es más específico o más fino que el otro).

Esto implica que una norma $\mathrm{N} 1$ al ser aplicada necesariamente entrará en conflicto con una norma N2, pero no necesariamente al aplicar N2 ello conllevará un conflicto con N1. En estos supuestos, $\mathrm{N} 2$ tiene un ámbito de aplicación más amplio que N1, pero todo el ámbito de aplicación de N1 está contenido en N2.

Formalmente, esto se representa como el conjunto de estas dos normas: (p->0q) y (p.r->0 $\neg q)$. Como podemos ver, entre ambas normas hay una propiedad compartida y otra que no, de forma que cada vez que se verifique la propiedad compartida y la no compartida (p.r) tendremos un conflicto normativo.

c) Conflictos parcial-parcial entre dos o más normas: en estos casos cada una de las normas posee ámbitos de aplicación diferentes, pero en caso que se verifiquen estas propiedades compartidas, entonces se produce un conflicto normativo. En líneas anteriores hemos visto este tipo de conflictos normativos al dar cuenta de los casos de conflictos de instanciación como un problema de imposibilidad lógica.

Formalmente, esto se representa como el conjunto de estas dos normas: (p->0q) y ( $\mathrm{r}->0\urcorner \mathrm{q})$. Como podemos ver, entre ambas normas no hay propiedades compartidas, de forma que cada vez que se verifiquen dichas propiedades no habrá

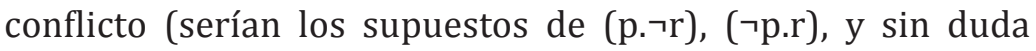


APUNTES CONCEPTUALES PARA LA IDENTIFICACIÓN...

( $\neg$ p. $\neg$ r)). Tendremos un conflicto normativo sólo en el caso que se verifiquen ambas propiedades de forma concurrente, es decir, que tengamos un caso (p.r).

En relación a esta clasificación de conflictos, Giovanni Battista $\operatorname{Ratti}^{48}$ ha formulado una precisión relevante. Atendiendo a la operatividad del refuerzo del antecedente todos los conflictos normativos, al momento de ser identificados, operan como un caso de conflicto total-total. En los tres casos de conflicto normativo tenemos un supuesto de inconsistencia en caso de verificarse una propiedad (sea esta compartida por los dos antecedentes en el supuesto de conflicto total-parcial, o por concurrir en un caso, en el supuesto de conflicto parcial-parcial). Siguiendo la representación empleada previamente, tendremos un conflicto cada vez que se verifique p.

En caso de conflicto total-parcial tenemos un conflicto entre N1 compuesta por (p->0q) y N2 compuesta por (p.r->0 ᄀq). En estos supuestos, en caso de presentarse la propiedad $r$, tenemos que hacer dos derivaciones aplicando el refuerzo del antecedente:

N1': p.r->0q

N2': p.r->0 $q \mathrm{q}$

En caso de conflicto parcial-parcial tenemos una situación similar ilustrada anteriormente. En estos casos tenemos un conflicto entre N3 compuesta por ( $p->0 q)$ y N4 compuesta por ( $r->0 \neg q)$. En estos supuestos, en caso de presentarse la propiedad p, tenemos que hacer dos derivaciones aplicando el refuerzo del antecedente:

$$
\begin{aligned}
& \text { N3': p.r->Oq } \\
& \text { N4': p.r->0ᄀq }
\end{aligned}
$$

En este sentido, al realizar una inferencia lógica de cada norma, tendremos que los casos de conflicto total-parcial y parcial-parcial son maneras alternativas de dar cuenta de un conflicto total-total. ${ }^{49}$

48

49

Ratti, Giovanni Battista, "Negation in legislation”, cit., p. 144.

En contraposición a esta conclusión, Navarro y Rodríguez sostienen que los 


\section{CONCLUSIONES}

Luego de describir y analizar las diferentes formas de justificar que dos normas están en conflicto entre sí, concluyo lo siguiente: en primer lugar, los juristas empleamos, de manera expresa o implícita, el principio lógico de no contradicción al momento de identificar una antinomia. Esto supone la necesidad de ser preciso al momento de justificar que estamos frente a un conflicto normativo: que el destinatario de la norma no puede satisfacer el consecuente de dos normas aplicables a un mismo caso individual.

En segundo lugar, los juristas empleamos, de manera expresa o implícita, la noción de refuerzo del antecedente al momento de: i) identificar el contenido de una norma; ii) determinar que una norma es aplicable a un caso individual, y iii) identificar un conflicto normativo. Esto supone que el desarrollo lógico de las normas es una operación necesaria para determinar la operatividad de las normas y para justificar, adecuadamente, que estamos frente a un caso de antinomia.

En tercer lugar, los conflictos normativos son, en términos precisos, de tipo total-total, entre: i) normas derivadas de norma expresa con normas derivadas de norma expresa; ii) normas derivadas de norma expresa con normas derivadas de norma implícita, o iii) normas derivadas de norma implícita con normas derivadas de norma implícita.

conflictos normativos solo pueden ser parciales. Estos autores, quienes entienden los conflictos normativos como casos de no posible cumplimiento de dos normas aplicables por imposibilidad lógica, sostienen que, si tomamos en cuenta las normas derivadas de cada norma, entonces tendremos fragmentos de la norma que no estará implicada en el conflicto. En este sentido, si inferimos las normas derivadas de cada norma analizada, vemos que solo en algunos casos habrá contradicción, pero habrá otros supuestos (otros casos inferidos) en los que no. Navarro, Pablo y Rodríguez, Jorge, "Entailed Norms and the Systematization of Law", cit., p. 104. Al respecto, considero que si el punto es aclarar qué casos entran en conflicto y porqué, entonces tenemos que analizar los casos que guardan imposibilidad lógica de mutuo cumplimiento. El resto de casos derivables de una norma genérica solo nos permiten aclarar otras posibilidades de aplicación, pero no dan cuenta de la relación entre estas dos normas en conflicto. Al respecto, ¿qué tipo de conflicto normativo es el caso de dos normas lógicamente incompatibles entre sí? Solo podría haber un conflicto total-total considerando el refuerzo del antecedente. 
En cuarto lugar, la justificación de que dos normas están en conflicto entre sí requiere asumir el principio lógico de no contradicción y la aplicación del refuerzo del antecedente. En efecto, las antinomias en el derecho son casos en los que dos normas condicionales prescriptivas entran en conflicto entre sí a mérito de la incompatibilidad lógica de sus normas derivadas. En otros términos, tras identificar el contenido de la norma (expresa o implícita) a través del empleo de métodos lógico-deductivos se verifica que dos normas derivadas comparten el mismo caso genérico, pero con consecuencias normativas contrarias o contradictorias entre sí, lo cual genera que para el destinatario sea imposible cumplir con las prescripciones contenidas en cada norma.

En quinto lugar, si la justificación de conflictos normativos requiere el uso (expreso o implícito) de conceptos lógicos, ello pone de relieve la necesidad de formar a los juristas en nociones básicas de lógica deóntica.

\section{REFERENCIAS}

Alchourrón, Carlos, “Conflictos entre normas y revisión de sistemas normativos", Análisis lógico y derecho, Madrid, CEC, 1991.

Alchourrón, Carlos y Bulygin, Eugenio, "Fundamentos pragmáticos para una lógica de normas", Análisis lógico y derecho, Madrid, CEC, 1991.

Alchourrón, Carlos y Bulygin, Eugenio, Sistemas normativos, Introducción a la metodología de las ciencias jurídicas, 2a. ed., Buenos Aires, Astrea, 2012.

Alchourrón, Carlos y Bulygin, Eugenio, “Norma jurídica”, en GARzón VALDEs, Ernesto y LAPORTA, Francisco (ed.), Enciclopedia Iberoamericana de filosofía, el derecho y la justicia, Madrid, Trotta, 2016.

Boвbio, Norberto, Contribución a la teoría del derecho, Madrid, Debate, 1990. 


\section{VÍCTOR GARCÍA YZAGUIRRE}

Bulygin, Eugenio, "Teoría y técnica de la legislación”, Análisis lógico y derecho, Madrid, CEC, 1991.

Chiassoni, Pierluigi, Técnicas de interpretación jurídica, breviario para juristas, Madrid, Marcial Pons, 2011.

Chiassoni, Pierluigi, Interpretation without Truth, a Realistic Enquiry, Cham, Springer, 2019.

GavazzI, Gioacomo, Delle antinomie, Turín, Giappichelli, 1959.

GUASTINI, Riccardo, Interpretar y argumentar, 2a. ed., Madrid, CEPC, 2014.

Hage, Jaap, "Rule Consistency”, Law and Philosophy, núm. 19, 2000.

ITURRALDE, Victoria, "Una aproximación al tema de las antinomias normativas: problemas relativos a la identificación de las mismas", Anuario de Filosofía del Derecho, núm. 4, 1987.

Lindahl, Lars y ReIDHAV, David, "Conflict of Legal Norms: Definition and Varieties", en Araszkiewicz, Michał y PŁEszka, Krysztof (eds.), Logic in the Theory and Practice of Lawmaking, Cham, Springer, 2015.

Martínez Zorrilla, David, "Conflictos normativos", en FABra ZAMORA, Jorge y NúÑEZ VAQUERO, Álvaro, Enciclopedia de filosofía y teoría del derecho, México, UNAM, 2015, vol. 2.

MazzAreze, Tecla, "Antinomie, paradossi, logica deontica", Rivista Internazionale di Filosofia del Diritto, vol. 61, núm. 4, 1984.

NAVARro, Pablo y Rodríguez, Jorge, Deontic Logic and Legal Systems, Cambridge, Cambridge University Press, 2014.

Navarro, Pablo y Rodríguez, Jorge, "Entailed Norms and the Systematization of Law", en Araszkiewicz, Michał y PŁeszKA, Krysztof (ed.), Logic in the Theory and Practice of Lawmaking, Cham, Springer, 2015.

ORunesu, Claudina, Positivismo jurídico y sistemas constitucionales, Madrid, Marcial Pons, 2012.

RATTI, Giovanni Battista, El gobierno de las normas, Madrid, Marcial Pons, 2013.

RATTI, Giovanni Battista, "Normative Inconsistency and Logical Theories: a First Critique of Defeasibilism", en ARASZKIEWICZ, Michał y ŠAVELKA, Jaromir, Coherence: Insight from 
APUNTES CONCEPTUALES PARA LA IDENTIFICACIÓN...

Philosophy, Jurisprudence and Artificial Intelligence, Dordrecht, Springer, 2013

RATTI, Giovanni Battista, "An Analysis of Some Juristic Techniques for Handling Systematic Defects in the Law", en BustamanTE, Thomas y DAHLman, Christian, Argument Types and Fallacies in legal argumentation, Dordrecht, Springer, 2015.

RATTI, Giovanni Battista, "Negation in legislation", en ARASZKIEWICZ, Michał y PŁeszKa, Krysztof (ed.), Logic in the Theory and Practice of Lawmaking, Cham, Springer, 2015.

RATTI, Giovanni Battista, "Normas jurídicas y contradicciones", en Redondo, María Cristina y Navarro, Pablo (ed.), La filosofía desde el derecho, homenaje a Ricardo Caracciolo, México, Fontamara, 2016.

RATTI, Giovanni Battista, "Rational Law-Giving: Structural and Interpretative Queries", Analisi e Diritto, 2017.

RATTI, Giovanni Battista y RodRíGUEz, Jorge, “On Coherence as a Formal Property of Normative Systems”, Revus, núm. 27, 2015.

RATTI, Giovanni Battista y RodríGUEz, Jorge, "Los principios fundamentales de la lógica en el derecho", Analisi e Diritto, 2018-1.

RoDRíGUEz, Jorge, “Contradicciones normativas: jaque a la concepción deductivista de los sistemas jurídicos", Doxa, vol. 17-18, 1995.

RoDríguEz, Jorge, Lógica de los sistemas jurídicos, Madrid, CEC, 2002.

RodríguEz, Jorge, "Sistemas normativos, lagunas jurídicas y clausura lógica”, Anuario de Filosofía del Derecho, 2015.

Ross, Alf, Sobre el derecho y la justicia, Buenos Aires, Eudeba, 1963.

Ross, Alf, Lógica de normas, Madrid, Tecnos, 1971.

Von Wright, George, "Is there a Logic of Norms?", Ratio Juris, vol. 4, núm. 3, 1991.

Zorzetto, Silvia, La norma speciale. Una nozione ingannevole, Pisa, ETS, 2010.

Zuleta, Hugo, Norma y Justificación, Madrid, Marcial Pons, 2008. 